\title{
EXPERIMENTAL STUDY OF THE FIELD IN THE GAP OF SYNCHRONOUS MACHINE WITH THE USE OF SINUSOIDAL TURNS
}

\author{
Mustafaquliva Gulzoda ${ }^{1}$, Maxmadiyev Giyos ${ }^{2}$, Zaripov Odiljon ${ }^{3}$, Zaripova Shaxlo ${ }^{4}$ \\ ${ }^{1}$ Professor, Tashkent State Technical University, Tashkent, Universitetskaya str. 2, Uzbekistan \\ ${ }^{2}$ Senior teacher, Tashkent State Technical University, Tashkent, Universitetskaya str.2, Uzbekistan \\ ${ }^{3}$ Senior teacher, Tashkent State Technical University, Tashkent, Universitetskaya str.2, Uzbekistan \\ ${ }^{4}$ Assistant, Karshi Engineering and Economics Institute, Kashkadarya region, Karshi, Mustaqillik ave., 225, Uzbekistan
}

\begin{abstract}
The paper deals with experimental study of the gap of a synchronous machine with using sinusoidal turns. The characteristics of a three-phase short circuit and the current dependence of the winding excitation are presented. Static modes of operation of synchronous machines in the stationary state of the rotor by supplying the stator and rotor windings with a direct current conducted by means of sinusoidal and cosine-shaped turns with respect to the transverse axis established in the air gap on the rotor surface along the longitudinal and transverse axes, respectively.
\end{abstract}

\section{Introduction}

In any electric machine, energy is converted from one type to another: in the generators, the mechanical energy communicated to the shaft of the electric machine is converted into electric energy, in the motors, the energy consumed from the power source is converted into mechanical shaft rotation energy.

Electric machines have the property of reversibility, that is, under certain conditions they can work both as electric power generators and as engines that drive various operating mechanisms, as well as in electromagnetic braking modes and some other special modes. However, the characteristics of various types of machines vary significantly. Synchronous Machine constitutes of both synchronous motors as well as synchronous generators. An alternating current (AC) system has some advantages over direct current (DC) system. Therefore, the AC system is exclusively used for generation, transmission and distribution of electric power [1]. The machine which converts mechanical power into AC electrical power is called as Synchronous Generator or Alternator. However, if the same machine can be operated as a motor is known as Synchronous Motor.

The rational use of electric $\mathrm{AC}$ machines in operation depends mainly on the accuracy of determining the characteristics and operational parameters in static and dynamic modes of their operation. The study of the properties of machines is carried out both in the field of theoretical current and in the field of experimental stresses. The accuracy of determining the parameters and characteristics of $\mathrm{AC}$ machines to a large extent depends on how fully the saturation of their magnetic circuits is taken into account when calculating or conducting an experiment. A more accurate analysis of various modes of operation of $\mathrm{AC}$ machines can be made only if the exact values of the parameters are substituted into their analytical expressions $[2,3]$.

\section{Investigation the parameters of $A C$ machines}

The parameters of $\mathrm{AC}$ machines with a saturated magnetic circuit are not unambiguous, but vary rather widely depending on their operating mode. They depend on the complex mutual influence of the component of magnetic fluxes actually existing in the machine and are complex functions of electrical quantities that determine the operating modes of the machine. Taking into account various phenomena that actually exist in the machine (saturation of the magnetic circuit, eddy currents in various massive metal parts, hysteresis, uneven air gap between the ferromagnetic cores of the stator and rotor, etc.) makes the task of analytical study of various operating modes of AC electric machines extremely difficult. Orientation only on analytical methods in solving this problem without a broadly set experimental study cannot give satisfactory results, since operational parameters of modern widely used machines differ significantly from the calculated ones. This is an imperfection of taking into account the aforementioned phenomena existing in the machine by today's calculation methods. For this reason, it is necessary to have the results of experimental data on existing AC machines of various capacities and designs, showing the dependence of the operating parameters of the machine on various factors [4$6]$.

When developing experimental methods and investigating $\mathrm{AC}$ machines, it is necessary to strive to maximize the approximation of the experimental 
conditions to different operating conditions, as well as to maximize the approximation of the experimental conditions to different operational modes, as well as to maximize the simplification of the experiments, which would be relatively easy to implement as at the factory test bench, and in operating conditions. Various experimental research methods have been developed and used in a number of works by researchers. Despite the successes of a number of researchers, the methodology for more accurately determining the operating parameters and characteristics of AC machines, taking into account the influence of various operating factors, needs further development. The behavior of a working electric AC machine substantially depends on the nature of the change and distribution of the field in the main air gap between the stator and rotor cores, as in this space the bulk of the energy of the electromagnetic field of the machine is accumulated and the main process of energy conversion in the machine occurs in this space [4].

The value of electromagnetic moment, one of the main operational parameters of an electric AC machine, is determined by the magnitude of the main EMF arising in the air gap. A more accurate experimental determination of the first spatial harmonic field of the air gap in order to determine further the electromagnetic power of $\mathrm{AC}$ machines is currently problematic, because nonsynchronous components of magnetic induction exist in the electromagnetic field of machine's air gap, which makes it difficult to determine the main synchronous field [5].

For harmonic analysis of the electromagnetic field in the air gap of an alternating current machine in static and transient modes of its operation, measuring turns of a sinusoidal shape are used $[2,4,5,7,8]$.

In the equipment of Siemens, a sinusoidal turn (coil) is inserted into the air gap to measure the first spatial harmonic field when calculating transients in turbo generators [5]. By studying the EMF induced in the measuring sinusoidal coils installed in the air gap of the turbo- and hydro generators in [2], it is indicated that in the sinusoidal coil distributed along the stator bore, the EMF is induced only from the spatial harmonic field, the period of which coincides with the wavelength of the superimposed sinusoidal coil.

\section{Description of experiment}

Experimental studies of the static modes of operation of synchronous machines in the stationary state of the rotor by supplying the windings of the stator and rotor with direct current were carried out using turns of sinusoidal and cosine forms relative to the transverse axis, installed in the air gap on the surface of the rotor along the longitudinal and transverse axes, respectively [8]. In [7], it is noted that the oscillation of the electromagnetic moment in static and transient modes of operation of an $\mathrm{AC}$ machine is carried out by directly measuring the EMF of the air gap using a sinusoidal coil. Studies of the features of the field created by the armature winding in the air gap of an alternating current machine showed that the spatial harmonic fields of the multiphase armature winding fed by the multiphase current, in contrast to the harmonic excitation fields of a synchronous machine, are not rotating relative to the winding that creates them, but are restored and summing them in a certain a range of the order of spatial distribution gives a picture of the scattering field of the armature winding along the tooth crowns $[9,10]$.

The distribution curve of the scattering field along the teeth crowns around the circumference of the air gap shows that it forms pairs of poles, the flow of each of which is coupled to a certain groove, the total current of which is nonzero and flows in the air gap in the radial and tangential directions, and part of it can penetrate the rotor body and interlock with the contours in it. The scattering flux along the tooth crowns, mating with a sinusoidal coil laid in the air gap, induces the main harmonic EMF in it, the vector of which, depending on the machine operating mode, is directed in a certain way with respect to the air gap EMF. As a result of this, a sinusoidal turn for the main harmonic field, laid along the stator bore, does not directly measure the EMF of the air gap at load. Therefore, the conclusions made in [2] regarding the fact that "measuring coils sinusoidal distributed along the stator bore with a wavelength equal to the wavelength of the investigated spatial harmonic field are spatial filters that highlight the corresponding spatial harmonic fields" should not be recovered [9] spatial harmonic field of the armature, but only to the harmonic field of the excitation winding of synchronous machines rotating together with the rotor.

In transient conditions, when the armature current can several times exceed its nominal value of the EMF induced in the sinusoidal coil from the scattering stream along the tooth crowns, it will significantly distort the measured EMF of the air gap. The computer calculations of the scattering flux along the teeth crowns penetrating the sinusoidal coil installed along the stator bore [3] showed that it has a rather large value, especially in machines with a small air gap. In open-pole synchronous machines, the magnitude of the scattering flux along the tooth crowns depends on the nature of the armature current. Sinusoidal turns should be laid where possible in those parts of the air gap where the scattering field over the tooth crowns is practically absent. This field decreases with distance from the surface of the stator bore, reaching almost zero at a distance equal to half the stator tooth pitch. In bipolar turbogenerators, the air gap is usually greater than half of the tooth pitch of the stator and therefore they have the possibility of placing sinusoidal turns in the air gap outside the range of the scattering field along the tooth crowns In machines with a small gap, this is not possible and taking into account the emf induced in the sinusoidal coil from the scattering flux along the tooth crowns can be calculated.

To verify the theoretical considerations regarding the formation of a scattering field along the crowns of the anchor teeth, as a sum of spatial harmonic in a certain range of their spatial distribution and penetration of sinusoidal measuring coils there built for the main harmonic field of the air gap and installed in the air gap in a synchronous explicit pole machine type MCA -72/4, while the air gap under the pole piece was increased from $0.76 \mathrm{~mm}$ to a uniform $3.2 \mathrm{~mm}$. Two sinusoidal coils for 
the main harmonic field with a distance between them in the radial direction equal to $\Delta \rho=1.8 \mathrm{~mm}$ were installed at one place along its circumference within one pole division. One of the sinusoidal turns was located on the surface of the stator bore and had a half-wave length equal to pole division, and the second with a half-wave length was installed closer to the rotor.

$$
\tau_{p}=\frac{\pi(D-2 \Delta \rho)}{2 p}, \text { where } D \text { is a stator bore diameter; }
$$

$p$ is the number of pairs of poles.

In Figure 1 the characteristic of a three-phase short circuit $\mathrm{I}_{\mathrm{K}}=f\left(i_{\mathrm{B}}\right)$ is depicted, as well as the dependences $\mathrm{U}_{\mathrm{s}}$ $=f\left(i_{\mathrm{B}}\right)$ and $\Delta \mathrm{U}_{\mathrm{s}}=f\left(i_{\mathrm{B}}\right)$ in this mode. Figure 2 shows the dependence of the voltage at the terminals of the armature $\mathrm{U}$ on the stator current $\mathrm{I}$, as well as the dependences $\mathrm{U}_{\mathrm{s}}=$ $f(\mathrm{I})$ and $\Delta \mathrm{U}_{\mathrm{s}}=f(\mathrm{I})$ when powered by a three-phase current from the stator and the open excitation winding. A comparison of the curves shows that the value of $\Delta \mathrm{U}_{\mathrm{s}}$ at the same values of the stator current in both experiments is almost the same. When the machine is excited only from the rotor side, i.e. in idle mode, the value of $\Delta U_{\mathrm{s}}$ was practically equal to zero.

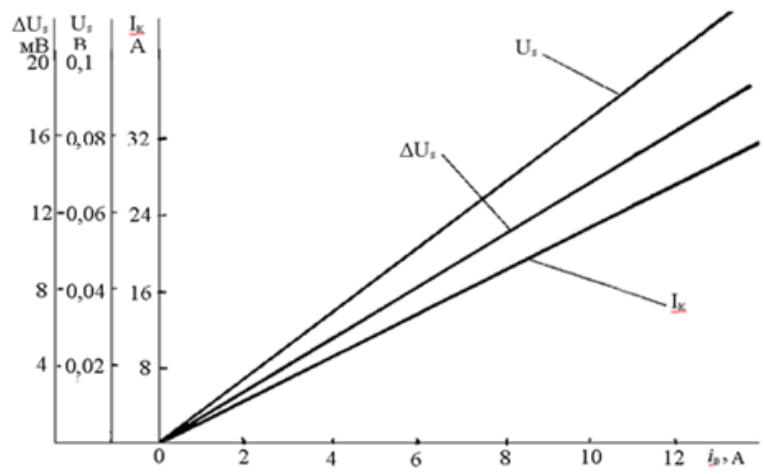

Fig.1. Three-phase short circuit characteristic and dependencies $\mathrm{U}_{\mathrm{s}}=f\left(i_{\mathrm{B}}\right)$ and $\Delta \mathrm{U}_{\mathrm{s}}=f\left(i_{\mathrm{B}}\right)$.

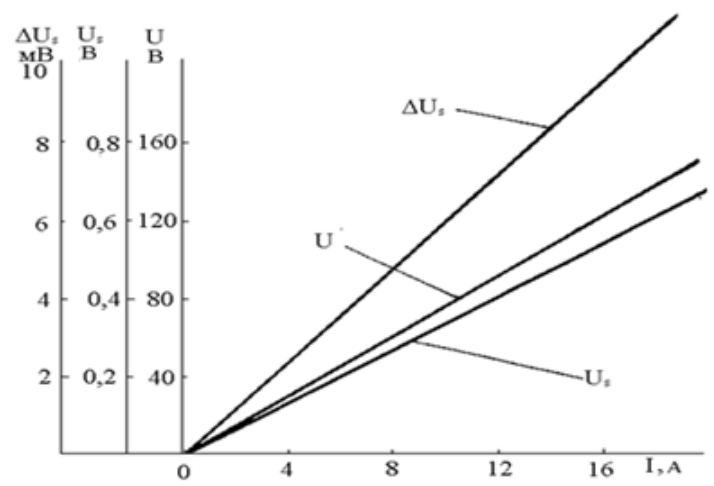

Fig. 2. Dependences $\mathrm{U}=f(\mathrm{I}), \mathrm{U}_{\mathrm{s}}=f(\mathrm{I})$ and $\Delta \mathrm{U}_{\mathrm{s}}=f(\mathrm{I})$ when powered through the stator.

\section{Concluding remarks}

As it is seen from the experimental results, each sinusoidal coil had a half-wave length equal to the distance between the axes of two adjacent poles, corresponding to its location in the radial direction of the air gap. Therefore, the value of $\Delta U_{\mathrm{s}}$ is mainly determined by the scattering stream of the armature along the tooth crowns, which penetrates the sinusoidal coil the more, the closer it is to the surface of the stator bore. This indicates that the harmonic field of the armature, in contrast to the harmonic field of excitation rotating together with the rotor, is recoverable and their combination in a certain range of a high order of spatial distribution forms a scattering field along the crowns of the teeth penetrating the sinusoidal coil, and rotating higher harmonics of the field of coil do not penetrate the sinusoidal coil for the fundamental harmonic.

The studies were conducted in idle, three-phase short circuit modes and when powered by a three-phase current separately from the stator with an open excitation winding. In all experiments, the rotation of the rotor with the synchronous speed of the measured EMF induced in the sinusoidal coil $\mathrm{U}_{\mathrm{s}}$ was supported, as well as the difference of the main harmonic EMF $\Delta \mathrm{U}_{\mathrm{s}}$ induced in the sinusoidal turns when they are reversed switched.

Thus, a sinusoidal coil cannot be used to directly measure the EMF of the air gap without taking into account the recovering nature of the harmonics of the armature winding field. This is especially important when studying transients associated with a large value of the armature current.

\section{References}

1.Hindmarsh J., Renfrew A., Electrical Machines and Systems/Newnes, imprint of Elsevier Science, 2002.

2.Bruskin, D.E. Electric machines. Part 2/ D.E. Bruskin, A.E. Zorokhovich, V.S. Khvost - M.: Alliance, 2016.-304 p.

3. Odilov G. Calculation of the scattering flux along the crowns of the teeth of the armature of an electric machine penetrating the sinusoidal coil// Proceedings of the $7^{\text {th }}$ International conference 'Theory and methods of calculating nonlinear circuits and systems'. - Tashkent, Tashkent State Technical University, 1995. p.62.

4.Odilov G. Investigation of the field in the gap of a synchronous machine using turns of a sinusoidal shape// Proceedings of Tashkent State Technical University "Actual issues in the field of humanitarian, socioeconomic and technical sciences", Tashkent. 1996. pp. $183-187$.

5. Hoshimov, F.A., Bakhadirov, I.I., Erejepov, M., Djumamuratov, B. (2019) Development of method for normalizing electricity consumption E3S Web Conf 139 doi:10.1051/e3sconf/201913901074

6. Hoshimov, F.A., Bakhadirov, I.I., Erejepov, M., Djumamuratov, B. (2019) Development of method for normalizing electricity consumption E3S Web Conf 139 doi:10.1051/e3sconf/201913901074

7.Baklin, V.S. Electric machines. Calculation of bipolar turbo generators. Textbook for Applied Baccalaureate/V.S. Bucklin. - Lyubertsy: Yurayt, 2016. $137 \mathrm{p}$.

8.Kopylov, I.P. Electric machines. Volume 1: Textbook for academic undergraduate/I.P. Kopylov. - Lyubertsy: Yurayt, 2016. - 267 p. 
9.Odilov $\mathrm{G}$. The recovering nature of the armature field in the air gap of a multiphase AC machine//Proceedings of Tashkent State Technical University "Actual issues in the field of humanitarian, socio-economic and technical sciences", Tashkent. 1996.pp. 57 - 61.

10.Odilov G. The scattering field on the crowns of armature teeth of an alternating current machine//Proceedings of the $7^{\text {th }}$ International conference 'Theory and methods of calculating nonlinear circuits and systems'. - Tashkent, Tashkent State Technical University, 1995. p. 63. 